\title{
Cosmic microwave background constraints on the strong equivalence principle
}

\author{
V. Boucher, ${ }^{1}$ J.-M. Gérard, ${ }^{1}$ P. Vandergheynst, ${ }^{2}$ and Y. Wiaux ${ }^{2}$, \\ ${ }^{1}$ Institut de Physique Théorique, Université catholique de Louvain, B-1348 Louvain-la-Neuve, Belgium \\ ${ }^{2}$ Signal Processing Institute, Swiss Federal Institute of Technology, CH-1015 Lausanne, Switzerland
}

(Dated: July 2004)

\begin{abstract}
We study the effect of a violation of the strong equivalence principle (SEP) on the cosmic microwave background (CMB). Such a violation would modify the weight of baryons in the primordial gravitational potentials and hence their impact in the establishment of the photon-baryon plasma acoustic oscillations before recombination. This cosmological Nordtvedt effect alters the odd peaks height of the CMB temperature anisotropy power spectrum. A gravitational baryonic mass density of the universe may already be inferred at the first peak scale from the analysis of WMAP data. Experimental constraints on a primordial SEP violation are derived from a comparison with the universe's inertial baryonic mass density measured either in a full analysis of the CMB, or in the framework of the standard big bang nucleosynthesis (BBN).

PACS numbers: 98.80.Es, 04.80.Cc, 98.70.Vc, 98.80.Ft
\end{abstract}

\section{INTRODUCTION}

The recent results of the cosmic microwave background (CMB) experiments, together with other cosmological tests, provide us today with a coherent picture of the structure and evolution of the universe. The corresponding canonical paradigm postulates a spatially flat universe which has undergone a period of inflation in its early ages. The present large scale structure of the universe essentially originates from primordial quantum energy density fluctuations around a homogeneous and isotropic background. These perturbations also left their imprint on the cosmic background radiation which decoupled from the rest of the universe some 380.000 years after the big bang. According to this cosmological model, the universe is filled in with about seventy percent of dark energy, twenty-five percent of cold dark matter, five percent of ordinary (baryonic) matter and a relic background of radiations. The recent one-year WMAP results led to an already precise determination of the corresponding cosmological parameters [1, 2]. However, in this context full credit may not be given to this analysis before the theoretical hypotheses on which the canonical paradigm is based are tested, notably through a thorough analysis of the CMB. Many questions may in fact be raised. The inflationary scenario and the structure of the initial conditions for energy density perturbations have been extensively analyzed (see for example [3, 4] for general considerations). Lately, the well established cosmological principle, postulating global homogeneity and isotropy of the universe, has also been challenged [4, 5, 6]. But, perhaps most fundamentally, one should question the theory of gravitation on which cosmology is developed, namely general relativity.

Any explicit theory of gravitation beyond general relativity introduces different effects which modify the char-

*Electronic address: yves.wiaux@epfl.ch acteristics of the CMB temperature anisotropy power spectrum. In particular the location and height of acoustic peaks should be altered proportionally to the strength of auxiliary gravitational couplings. This analysis has already been performed for pure Brans-Dicke theories 7] as well as generalized versions [8, 9, 10], leading to constraints on a possible scalar coupling. However these bounds do not take into account possible effects induced by the violation of the strong equivalence principle (SEP). This principle, essential feature of the theory of general relativity, notably postulates the constancy of the newtonian gravitational coupling $G$ in space and time. It distinguishes Einstein's theory from other metric theories of gravitation. Any SEP test therefore challenges general relativity in its most fundamental structure. The purpose of this article is to study the influence of a possible SEP violation on the CMB temperature power spectrum. In order to single out the generic effect of such a SEP violation on the $\mathrm{CMB}$, our approach naively conserves Einstein's equations for the gravitational field. Hence, under the hypothesis of the cosmological principle, the Friedmann-Lemaitre background and perturbed cosmological evolution remains unchanged. In this way, we avoid the introduction of multiple effects which could unnecessarily complicate the conceptual analysis. The SEP violation is simply introduced through the break down of energy-momentum conservation for compact bodies. The breaking term in the corresponding covariant equations depends on the gradient of the gravitational coupling $G$ with respect to spacetime coordinates.

In section 1 we introduce the SEP and its violation. Section $\amalg$ is devoted to the analysis of the effect of a SEP violation on the CMB temperature power spectrum. The SEP violation affects the weight of baryons in the primordial gravitational potentials and hence their impact in the establishment of acoustic oscillations of the plasma before the last scattering of photons. The odd peaks height enhancement of the CMB temperature power spectrum depends indeed on a gravitational baryonic mass density of the universe, not on its inertial baryonic mass density. 
We also discuss the characteristic amplitude of this effect and the uniqueness of its signature relatively to the variation of the canonical cosmological parameters. In section IV] we derive experimental constraints on the SEP violation. A gravitational baryonic mass density of the universe is inferred at the first peak scale from WMAP data. The constraints on the SEP violation are obtained from the measurement of the inertial baryonic mass density, either in a full analysis of the CMB temperature power spectrum, or through the independent determination of light element abundances in the framework of standard BBN. We discuss the proposed constraints, and finally conclude.

This article pursues the work done in 11], on the ground of both theory and data analysis.

\section{SEP VIOLATION}

The equivalence principle is an important fundament of any theory of gravitation. It is however implemented at different levels in different theories. The Einstein equivalence principle postulates the universality of free fall of test-bodies at one given point of a gravitational field (called weak equivalence principle), as well as the independence of the result of any non-gravitational experiment in a freely falling frame relative to the velocity of free fall and relative to where and when in the universe it is performed. A metric theory of gravitation postulates the geodesic motion of test-bodies, as well as the agreement of the results of any non-gravitational experiment performed in free fall with the laws of special relativity. By definition, all metric theories of gravitation therefore respect the Einstein equivalence principle. Technically, a theory of gravitation respects this principle if the Lagrange density for matter only depends on the matter fields and the spacetime metric, but not on possible auxiliary gravitational fields which directly couple to the metric. This structure implies indeed the general covariant conservation equations $T^{\mu \nu}{ }_{1 \nu}=0$ for the energy-momentum tensor $T^{\mu \nu}$, from which readily follows geodesic motion. On the other hand, nongravitational interactions are coupled to the metric field through the connexion, and therefore reduce to their special-relativistic structure in free fall. Both postulates of the Einstein equivalence principle are therefore ensured.

The strong equivalence principle extends the universality of free fall to compact bodies. By compact body, one means a body with a non-negligible amount of internal gravitational binding energy. It also extends to gravitational experiments the independence of the result of any experiment in free fall relative to the velocity of free fall and relative to where and when in the universe it is performed. The mere existence of an auxiliary field of gravitation coupled to the metric field violates this principle. The reason for this holds in the fact that it is not possible to cancel the effect of auxiliary (typically scalar or vector) fields by a local coordinate transformation, like it is for the tensor metric field. Auxiliary couplings will inevitably modify the result of gravitational experiments (and notably the structure of compact bodies) performed in a freely falling frame, therefore violating the SEP. The gravitational coupling $G$ itself will depend on the spacetime point through a dependence in the auxiliary fields, which implies by definition to a SEP violation. Aside from the Nordström scalar theory, only general relativity incorporates the equivalence principle at the level of the SEP [12, 13]. Testing the SEP violation is therefore a way of discriminating general relativity from other metric theories of gravitation such as extended Brans-Dicke or vector-tensor theories of gravitation.

If the newtonian gravitational coupling is a function of the position $x$ in spacetime, $G \rightarrow G(x)$, the mass $m$ of a compact body also depends on the position through its internal gravitational binding energy. An effective action for the geodesic motion of compact bodies may therefore be defined as: $S_{m a t}=-c \int m(x) d s$. Energy-momentum conservation is therefore broken through the introduction of a source term in the general covariant conservation equations. We adopt the corresponding expression as our mathematical implementation of a possible SEP violation:

$$
T^{\mu \nu}{ }{ }_{\nu}=G^{\mu} \frac{\partial T}{\partial G},
$$

where $T$ is the trace of the energy-momentum tensor. The dependence of the newtonian gravitational coupling on the spatial position $\vec{x}$ is parametrized through the relation $G(\vec{x})=G_{0}\left(1+\eta_{g} V(\vec{x}) / c^{2}\right)$, where $V(\vec{x})$ stands for the gravitational potential at the point considered, $G_{0}$ is the background value of the newtonian gravitational coupling in the absence of this potential, and $\eta_{g}$ is the parameter which defines the amplitude of the SEP violation. One may also define the compactness $s$ of a body as the sensitivity of its mass relative to $G$. It is equivalently given by the ratio of its internal gravitational binding energy $E_{g}$ to its total mass energy: $s=-d \ln m / d \ln G=\left|E_{g}\right| / m c^{2}$. The acceleration $\vec{a}$ of a body in a gravitational field now explicitly depends on its proper sensitivity $s$. This establishes the SEP violation through the so-called Nordtvedt effect [14, 15]. From the definition (11) indeed, we get in the non-relativistic (called quasi-newtonian) approximation: $\vec{a}=\vec{g}\left(1-\eta_{g} s\right)$, where $\vec{g}=-\vec{\nabla} V(\vec{x})$. In other words, the SEP violation induces a reduction (for $\eta_{g}>0$ ) or increase (for $\eta_{g}<0$ ) of the gravitational mass $m_{g}$ of a body relative to its inertial mass $m$, proportionally to its own compactness:

$$
m_{g}=m\left(1-\eta_{g} s\right) .
$$

From the experimental point of view, tests of the weak equivalence principle date back to Newton and its pendulum experiments. First tests of the SEP have been introduced several decades ago with the Lunar Laser Ranging experiment (see 16] for an extended review and references). This test still gives the best constraint on the 
parameter $\eta_{g}$ today $^{1}$ :

$$
\eta_{g}^{0} \leq 1 \times 10^{-3}
$$

with involved compactnesses of order $10^{-10}$ 16, 17]. A better constraint may be inferred in the framework of peculiar scalar-tensor theories of gravitation though. Indeed, the SEP violation actually introduces a new charge of gravitation beyond the mass, in terms of the compactness $s$. This new charge not only modifies the motion of compact bodies (Nordtvedt effect) but affects the dynamical structure of the corresponding theory, inducing potentially dominant dipole gravitational radiations associated with auxiliary fields of gravitation (scalar or vector). The analysis of the orbital period decrease rate of asymmetric binary pulsars is an extremely good probe of dipole radiations. The recent and unique measurement of the orbital decrease in such a binary, the neutron star - white dwarf PSR J1141 - 6545 [18, 19] gives a tight constraint on a scalar gravitational coupling. In pure Brans-Dicke theories (BD), the corresponding bound on the SEP violation inferred from [12, 18] reads: $\eta_{g}^{0(B D)} \leq 2.7 \times 10^{-4}$ (see also [20]).

\section{SEP AND CMB}

\section{Qualitative analysis}

In the primordial universe the photon gas is rather tightly coupled to electrons through Compton scattering. The electrons are themselves linked to protons through the Coulomb interaction. We may then consider a photon-baryon plasma in evolution in gravitational potentials. These gravitational potentials are essentially produced by the dominant cold dark matter component of the universe. About 380.000 years after the big bang, the temperature of the expanding universe had decreased too much to longer maintain hydrogen dissociation. The cosmic microwave background radiation observed today corresponds to a snapshot of this photon gas which decoupled from the rest of the universe at the time of last scattering. The anisotropy distribution on the sky today is determined by the multiple physical phenomena which governed the evolution of the plasma before recombination, and therefore contains all the information on the structure and evolution of the universe (defined in terms of cosmological parameters). The plasma underwent oscillations, responsible for relative temperature fluctuations in the associated black-body spectrum. In the corresponding angular power spectrum, this oscillation process translates into a series of acoustic peaks at scales smaller than the horizon size at last scattering.

\footnotetext{
${ }^{1}$ Quantities measured at the present epoch are indexed by the superscript ${ }^{0}$.
}

Odd peaks correspond to scales which had reached maximum compression (rarefaction) at the time of last scattering in potential wells (hills). Even peaks correspond to maximum rarefaction (compression) in potential wells (hills). The general shape of this spectrum therefore exhibits a Sachs-Wolfe plateau at scales beyond the horizon size at last scattering, followed by the acoustic peak series under the horizon size. Notice that, up to now, the standard CMB analysis has been based on the study of the precise characteristics of the temperature anisotropy angular power spectrum. The cosmological parameters are determined through a best fit of the theoretical cosmological models with experimental data (see notably [1, 2] for the WMAP analysis).

The oscillations of the plasma are electromagnetic acoustic oscillations of the photon gas. However, the action of gravity is introduced through a purely newtonian coupling of the baryonic content of the plasma to the dark matter potentials. The effect of this coupling is to shift the zero point (equilibrium) of the oscillations toward more compressed states in potential wells, and rarefied states in potential hills. Consequently, the height of odd peaks relative to even peaks is enhanced proportionally to the total baryon weight in the dark matter potentials [21, 22, 23, 24, 25].

If the SEP is violated through a spatial dependence of the newtonian gravitational coupling (Nordtvedt effect), gravitational masses differ from inertial masses. The temperature power spectrum peaks height therefore bears the imprint of a possible SEP violation as it essentially originates from a gravitational interaction and therefore depends on a gravitational baryonic mass density:

$$
\left(\rho_{b}\right)_{g}=\rho_{b}\left(1-\eta_{g} s_{b}\right)
$$

rather than on the inertial baryonic mass density $\rho_{b}$. The compactness $s_{b}$ must be associated with a baryon-region seen as a compact body at the relevant cosmological scale.

\section{Plasma evolution equations and SEP violation}

The purpose of this subsection is to derive more technically the main result of the last subsection. The evolution equations for the photon-baryon plasma in the tight coupling limit are derived from the generalized covariant energy-momentum tensor equations (1).

The tight coupling limit amounts to consider an infinite Compton interaction rate which implies the equality of the mean photon and baryon velocities: $\vec{v}_{\gamma}=\vec{v}_{b}$. In this standard approximation, the photon-baryon gas may be entirely described as a fluid with the energymomentum tensor $T^{\mu \nu}=\left(\rho+P / c^{2}\right) u^{\mu} u^{\nu}-P g^{\mu \nu}$. The equation of state relating pressure and density reads: $P=\lambda \rho c^{2}$, with $\lambda=0$ for matter and $\lambda=1 / 3$ for radiation. Restricting ourselves to a flat universe, in the newtonian gauge, with conformal time $\eta$ and comobile coordinates $\vec{x}$, we may write the perturbed spacetime metric as $g_{00}(\vec{x}, \eta)=a^{2}(\eta)\left(1+2 \Psi(\vec{x}, \eta) / c^{2}\right), g_{0 i}(\vec{x}, \eta)=0$, 
and $g_{i j}(\vec{x}, \eta)=-\delta_{i j} a^{2}(\eta)\left(1+2 \Phi(\vec{x}, \eta) / c^{2}\right)$. The factor $a(\eta)$ stands for the scale factor of the expanding universe normalized to its present size $\left(a^{0}=1\right)$. The scalar perturbations $\Psi(\vec{x}, \eta)$ and $\Phi(\vec{x}, \eta)$ may been seen as newtonian potentials.

From the equations (11) we readily obtain the continuity and Euler equations for the fluid under consideration. In the Fourier space, to first order in the relative density perturbations $\delta(\vec{k}, \eta)$, comobile velocity $v(\vec{k}, \eta)$, and gravitational potentials $\Psi(\vec{k}, \eta)$ and $\Phi(\vec{k}, \eta)$, these equations read respectively:

$$
\begin{aligned}
\dot{\delta}= & -(1+\lambda)\left(i \vec{k} \cdot \vec{v}+3 \frac{\dot{\Phi}}{c^{2}}\right)-\left(1-3 \frac{c_{s}^{2}}{c^{2}}\right) s \frac{\dot{G}}{G} \\
\dot{\vec{v}}= & -\frac{\dot{a}}{a}\left(1-3 \frac{c_{s}^{2}}{c^{2}}\right) \vec{v}-i \vec{k}\left[c_{s}^{2} \frac{\delta}{1+\lambda}\right. \\
& \left.+\Psi\left(1-\left(1-3 \frac{c_{s}^{2}}{c^{2}}\right) \frac{\eta_{g} s}{1+\lambda}\right)\right] .
\end{aligned}
$$

Dotted variables here stand for their derivative with respect to the conformal time. The sound speed in the fluid $c_{s}$ and the compactness $s$ characterizing a given fluid volume are background space-independent quantities. The $s$-terms represent the explicit modification due to SEP violation of the canonical [23, 24, 25] evolution equations for a single component fluid.

In order to find the evolution equations for the photons, we just apply this set of equations to a photon fluid with $\vec{v}=v_{\gamma} \hat{k}$, taking into account the presence of baryons in the sound speed and the compactness. The sound speed reads $c_{s}^{2}=d P_{\gamma} / d\left(\rho_{\gamma}+\rho_{b}\right)=c^{2} / 3(1+R)$, where $\rho_{b}$ and $\rho_{\gamma}$ are respectively the background inertial baryonic mass density and photon density of the universe, and $R=3 \rho_{b} / 4 \rho_{\gamma}$ is the canonical normalization of the baryonic mass density by the photon density. The photon gravitational binding energy is negligible and the fluid compactness reduces to the baryonic component $s_{b}$, which is studied in the next subsection. The fluid density and velocity may be expressed in terms of the monopole and dipole moments $\Theta_{0}(\vec{k}, \eta)$ and $\Theta_{1}(\vec{k}, \eta)$ of the photon relative temperature distribution: $\delta_{\gamma}(\vec{k}, \eta)=4 \Theta_{0}(\vec{k}, \eta)$ and $v_{\gamma}(\vec{k}, \eta)=-3 i \Theta_{1}(\vec{k}, \eta)$. In this context, the plasma evolution equations for $X(\vec{k}, \eta)=\Theta_{0}(\vec{k}, \eta)+\Phi(\vec{k}, \eta) / c^{2}$ and $\Theta_{1}(\vec{k}, \eta)$ read:

$$
\begin{aligned}
\ddot{X}+\frac{\dot{R}}{1+R} \dot{X}+k^{2} c_{s}^{2} X= & k^{2} c_{s}^{2}\left[\frac{\Phi}{c^{2}}\right. \\
& \left.-\frac{\Psi}{c^{2}}\left(1+R\left(1-\eta_{g} s_{b}\right)\right)\right](7) \\
k \Theta_{1}= & -\dot{\Theta}_{0}-\frac{\dot{\Phi}}{c^{2}} .
\end{aligned}
$$

We do not consider here the term with temporal dependence of the newtonian coupling, though it would be worth analyzing its effect. Only the spatial dependence of $G$ is considered by analogy with the Nordtvedt effect.
The first equation sets the dynamics for damped oscillations for $\Theta_{0}$ with a forcing term (right-hand side). We clearly identify that the effect of baryons in this forcing term depends indeed on the gravitational baryonic mass density

$$
R_{g}\left(s_{b}, \eta_{g}\right)=R\left(1-\eta_{g} s_{b}\right),
$$

function of the compactness $s_{b}$, rather than on the inertial baryonic mass density.

Notice that in the limit of constant newtonian potentials $^{2} \Psi=\Psi^{*}, \Phi=\Phi^{*}$, with $R=R^{*}$, equation (7) reduces, for the effective temperature perturbation $Y=$ $\Theta_{0}+\Psi^{*} / c^{2}$, to $\ddot{Y}+k^{2} c_{s}^{2} Y=-k^{2} c_{s}^{2} R_{g}^{*}\left(s_{b}^{*}, \eta_{g}^{*}\right) \Psi^{*} / c^{2}$. The forcing term clearly reduces to the (quasi-)newtonian interaction between the baryons and the surrounding constant potentials. In the further approximations $s_{b}=s_{b}^{*}$ and $\eta_{g}=\eta_{g}^{*}$ discussed in the following, the interaction term is constant. We therefore recover the exact limit in which a constant zero-point shift of the acoustic oscillations originates the odd peaks height enhancement of the temperature power spectrum. But the acceleration of baryons is now a function of the compactness of the baryon-region considered. Equation (9) is therefore the mathematical expression of the cosmological Nordtvedt effect discussed in the former qualitative analysis.

\section{Compactness of baryon-regions}

Under the hypothesis of the cosmological principle, we live in a globally homogeneous and isotropic universe. As suggested in our qualitative analysis, let us consider a homogeneous spherical baryon-region of radius $L$ and total mass $M_{b}$. Its compactness calculated, in the spirit of the quasi-newtonian approach introduced in section III as the ratio of the internal gravitational binding energy over the total mass energy reads: $s_{b}=$ $3 G M_{b} / 5 L c^{2}=4 \pi G \rho_{b} L^{2} / 5 c^{2}$. The mean baryon density scales as $\rho_{b}(a)=\rho_{b}^{0} a^{-3}$. At each instant in the course of the universe expansion, the maximum size of the radius $L$ is set by the event horizon: $L_{1}(\eta)=c a \eta$. This hypothesis is natural as the event horizon defines at each moment the maximal distance through which particles may have interacted gravitationally since the primordial ages of the universe (after inflation), and therefore the maximal size of a body. In matter and radiation universes, the Friedmann-Lemaître equations (in the considered limit where Einstein equations are preserved) determine the evolution of the scale factor with time as $\eta / \eta^{0}=a^{1 / 2}$ and $\eta / \eta^{0}=a$, respectively.

The compactness of a baryon-region therefore grows linearly with the scale factor in a radiation era, while it is constant in a matter era. Recombination takes place

\footnotetext{
${ }^{2}$ Quantities measured at recombination are indexed by the superscript * .
} 
after the matter-radiation equilibrium, inside the matter era. For the sake of the analogy with the Nordtvedt effect on compact bodies in a gravitational field, we consider in the following a constant compactness over the course of the universe evolution until recombination. It is evaluated at its value in the matter era, say at last scattering $\left(s_{b}=s_{b}^{*}\right)$. The low baryon density turns out to be largely compensated by the considered cosmological scales to give a non-negligible contribution to the compactness. In terms of physical quantities (the Hubble constant, the age of the universe and the relative baryon density), we get a compactness

$$
s_{b}^{1 *}=\frac{27}{10}\left(H^{0} t^{0}\right)^{2} \Omega_{b} \simeq 0.1,
$$

for the maximal radius $L_{1}$. This compactness is the sensitivity to be considered at the scale of the wavelength $\lambda_{1}$ associated with the first acoustic peak. The sensitivity of the baryonic body relevant for the subsequent acoustic peaks $\left(\lambda_{n}\right)$ scales like $n^{-2}$ since the compactness $s_{b}$ of the baryon-region considered is proportional to the square of its radius $L$ :

$$
s_{b}^{n *} \simeq 0.1 n^{-2}
$$

Let us now briefly comment on the implications of these results.

\section{Amplitude of the SEP violation effect}

The value $s_{b}^{1 *} \simeq 0.1$ in (10) implies that a SEP violation parameter of order unity at the time of recombination, $\eta_{g}^{*} \simeq 1$, would affect the first peak height by $10 \%$ (see equation (9)). In present CMB analyses, the cosmological parameter $\Omega_{b} h^{2}$ identifying the baryon content of the universe is essentially extracted from the measurement of the relative height between the first and second peaks of the temperature angular power spectrum. In this regard, it measures the gravitational, rather than inertial, baryonic mass density of the universe. The recent one-year WMAP analysis gives this parameter with a precision of $4 \%$. Consequently, the present CMB data will already allow us to derive interesting constraints on a possible SEP violation.

\section{Uniqueness of the SEP violation signature}

The peculiar $n^{-2}$ scaling of the baryon-regions compactness $s_{b}^{n *}$ in (11) ensures the orthogonality of the SEP violation signature relative to the effect other cosmological parameters on the CMB temperature angular power spectrum. The signature of the SEP violation may indeed be disentangled from the effect of other parameters through the corresponding $n^{-2}$ scaling of the odd peaks height. The measurement of the SEP violation parameter $\eta_{g}^{*}$ at recombination is therefore in principle possible, simultaneously to the determination of the canonical
[21, 26] cosmological parameters. The Planck satellite is designed to achieve a better sensitivity in the temperature anisotropies measurement, as well as a better resolution on the sky, than the present WMAP mission. This mission will notably give access to the whole series of acoustic peaks in the temperature anisotropies angular power spectrum [3], therefore allowing an unambiguous analysis of a possible SEP violation.

\section{EXPERIMENTAL CONSTRAINTS}

In this section, we establish experimental constraints on the SEP violation parameter $\eta_{g}^{*}$ at recombination, and discuss their significance in comparison with existing bounds at our epoch and theoretical predictions at the exit of the radiation era.

A precise analysis of a possible SEP violation must be performed through a best fit of our modified theory (11) and experimental data, taking into account the substitution (9) in the plasma evolution equations before recombination. Here, we determine bounds on a possible SEP violation by the analysis of the one-year WMAP experimental error bars on the observables of interest. This simple approach finds its justification in the fact that our modified theory assumes the cosmological Nordtvedt effect to be the only perturbation to the cosmic background anisotropy spectrum relatively to the canonical paradigm based on general relativity. In a first approach, one can determine the gravitational and inertial baryonic mass densities of the universe at recombination from their specific (orthogonal) signatures on the CMB power spectrum characteristics. A second generic approach consists in determining the gravitational baryonic mass density through the analysis of the CMB, using as a prior the measurement of the inertial baryonic mass density by independent observations. In that respect, we will consider here the determination of the inertial baryonic mass density through the measurement of light element abundances in the framework of standard BBN.

\section{CMB-CMB constraint}

The baryon content of the universe affects the CMB temperature power spectrum in different ways. The major effect is a dependence of the odd peaks height due to the weight of baryons in the surrounding gravitational potentials. We already know that this effect is actually a function of a gravitational baryonic mass density $R_{g}^{*}$. It bears the imprint of a possible SEP violation in terms of the already discussed $n^{-2}$ scaling. This unique signature adds to the canonical odd peaks height enhancement related to the inertial baryonic mass density $R^{*}$ (equation (9)). But any increase of the baryon density also naturally induces a decrease of the sound speed for the propagation of the acoustic oscillations in the primordial plasma, therefore affecting the peaks location, rather 
than their height. Increasing the baryon density also decreases the diffusion length, defined as the scale below which inhomogeneities are damped because of the finite Compton interaction rate. These last two effects are related to electromagnetic (rather than gravitational) phenomena and are consequently independent of the SEP violation. They only depend on the inertial baryonic mass density $R^{*}$.

As already mentioned, the forthcoming Planck mission will probe all these signatures. At present however, the temperature power spectrum characteristics are known with precision only up to the second peak through the one-year WMAP data. It is therefore rather difficult to disentangle a SEP violation from variations of other cosmological parameters, notably from $R^{*}$. However, assuming that all parameters, other than $R_{g}^{*}$ and $R^{*}$, are fixed to their accepted value, we may infer a constraint on $\eta_{g}^{*}$. On the one hand, we consider the one-year WMAP value of the cosmological parameter $\Omega_{b} h^{2}$ as a measure of the relative height between the first and second peaks [1], hence originating from the gravitational baryonic mass density $R_{g}^{*}\left(s_{b}^{1 *}, \eta_{g}^{*}\right)$, at last scattering, and at a scale corresponding to the maximum oscillation wavelength. On the other hand however, the specific analysis of the first peak position gives the inertial baryonic mass density $R^{*}$, through the dependence of the peaks location in the sound speed in the primordial plasma. A simple analysis of the one-year WMAPext (i.e. WMAP extended to the CBI and ACBAR experiments [1, 2]) error bars on these two observables gives the bound: $\left|\eta_{g}^{*} s_{b}^{1 *}\right| \leq 0.06$. From the estimated value (10) for $s_{b}^{1 *}$, we readily obtain the following constraint on the SEP violation in terms of $\eta_{g}^{*}$ :

$$
\left|\eta_{g}^{*(C M B)}\right| \leq 0.6
$$

\section{CMB-BBN constraint}

The standard BBN model may also infer the inertial baryonic content of the universe from the determination of light element $\left(\mathrm{D},{ }^{3} \mathrm{He},{ }^{4} \mathrm{He},{ }^{7} \mathrm{Li}\right)$ abundances. These abundances are studied in low-metallicity systems in such a way that they still significantly reflect primordial quantities. In this context, the baryon content of the universe is usually quoted in terms of $\Omega_{b} h^{2}$, rather than $R^{*}$. Notice that BBN is also affected in the framework of a specific alternative theory of gravitation [8, 27, 28]. However it is independent of the SEP violation considered here.

The primordial ${ }^{4} \mathrm{He}$ abundance is determined to better accuracy than in the case of other light elements 29, 30, 31]. However, it is rather insensitive to the baryon content. The measurement of ${ }^{4} \mathrm{He}$ abundance therefore has to be extremely precise if one wants to obtain a small uncertainty on $\Omega_{b} h^{2}$ or $R^{*}$. The most recent estimate, obtained in the analysis of dwarf irregular and compact blue galaxies gives, for the ${ }^{4} \mathrm{He}$ mass fraction: $Y_{p}=0.2421 \pm 0.0021$ 29]. In the framework of the standard BBN theory, the corresponding baryon content is $\Omega_{b} h^{2}=12_{-2}^{+3} \times 10^{-3}$ or $R^{*}=0.334_{-0.056}^{+0.084}$. The baryon density inferred from the primordial lithium-tohydrogen abundance ratio ${ }^{7} \mathrm{Li} / \mathrm{H}$ lies around the same values [32, 33, 34]. The one-year WMAPext value, still understood as a measurement of the relative height of the first two peaks of the CMB temperature power spectrum, gives a significantly higher value for $R_{g}^{*}$ : $\Omega_{b} h^{2}=$ $(22 \pm 1) \times 10^{-3}$ [2], or $R_{g}^{*}=0.613 \pm 0.028$. The confrontation of these numbers would, in our approach based on (9), suggest a rather high negative value for the parameter $\eta_{g}^{*}$. In other words, assuming that the ${ }^{4} \mathrm{He}$ and ${ }^{7} \mathrm{Li}$ analyses really reflect the baryon content of the universe, the gravitational interaction heavily violates the SEP, at least at the epoch of last scattering, if the whole discrepancy is accounted for by this effect. This would be the first experimental evidence that general relativity is not the correct theory of gravitation. However, large systematic uncertainties affect the ${ }^{4} \mathrm{He}$ and ${ }^{7} \mathrm{Li}$ abundance estimation. These may be related to observation or due to the lack of understanding of the complex physics in the evolution of these abundances 29, 34, 35]. Errors and incompletenesses in the standard BBN scheme may also lead to deviations [36, 37, 38]. Many efforts are made to reduce these systematic errors.

The deuterium abundance is extremely sensitive to the primordial baryon content. Moreover it may have been produced in significant quantities only during the BBN. Its measurement in quasar absorption line systems is therefore an extremely good probe of the baryon content of our universe [39, 40, 41, 42]. The most recent estimate of the primordial deuterium-to-hydrogen abundance ratio $D / H$ based on a recent analysis toward five quasars gives $D / H=2.78_{-0.38}^{+0.44} \times 10^{-5}[39]$. This value corresponds to a weighted average of the results obtained for each quasar independently. The corresponding value for the baryon content, in the framework of the standard BBN theory, reads $\Omega_{b} h^{2}=(21.4 \pm 2) \times 10^{-3}$, or $R^{*(B B N-D)}=0.596 \pm 0.056$. Combined with the oneyear WMAPext value given here above, this measure gives the following constraint on a possible SEP violation: $-0.14 \leq \eta_{g}^{*} s_{b}^{1 *} \leq 0.08$. This bound, once translated into a constraint on the parameter $\eta_{g}^{*}$, leads to:

$$
\eta_{g}^{*(B B N-D)}=-0.3 \pm 1 .
$$

The determination of the primordial ${ }^{3} \mathrm{He}$ abundance is more difficult as its destruction and production in stars are not well understood. However a recent upper limit on ${ }^{3} \mathrm{He} / \mathrm{H}$ leads to a prediction for the baryon content of the universe in complete agreement with deuterium measurements 43. Remains to be noticed that the dispersion of the values obtained for the deuterium abundance from different quasar absorption lines is bigger than expected from individual measurement errors. This dispersion could be real but the hypothesis of underestimated systematic errors in the measurements is favored [39, 40, 44]. More data would be needed to confirm the measurements and limit systematics. However, in the framework of light element abundance measurements, 
deuterium analysis remains the most reliable evaluation of the universe's baryon content thanks to its high sensitivity to the baryon content and the relative absence of deuterium production after BBN. In this context, the discrepancy between the baryon content inferred from $D$ and from ${ }^{4} \mathrm{He}$ or ${ }^{7} \mathrm{Li}$ analyses should be resolved by a better assessment of the systematics affecting the measurements of the last two elements abundances.

New physical scenarios beyond the standard BBN are also considered for solving this apparent tension. Leaving aside the present discrepancies among the BBN measurements, several proposals have recently been made for reconciling $\mathrm{BBN}$ and $\mathrm{CMB}$ measurements. The new physical effects invoked notably consider the modification of the number of relativistic particle species, variations of the strength of gravity in the early universe, or its dependence on the nature of interacting particles [45, 46, 47, 48, 49, 50]. Our last constraint on $\eta_{g}^{*}$ may be understood as an alternative solution in this direction.

\section{Discussion}

First we emphasize that accurate constraints on a primordial SEP violation should be determined by a best fit of our modified theory with experimental data. However, the numerical compatibility of the two independent bounds obtained, (12) and (13), supports our results. Also notice that in the framework of a specific alternative to general relativity the SEP violation is not the only new effect. The introduction of auxiliary gravitational fields affects the structure of gravitation itself and notably leaves signatures in the CMB as well as in the BBN. This will inevitably modify our bounds. In such a framework, the corresponding bounds on $\eta_{g}^{*}$ could also be run backward or forward over cosmological timescales for comparison, either with theoretical predictions on initial conditions $\left(\eta_{g}^{i}\right)$, or with present experimental constraints $\left(\eta_{g}^{0}\right)$.

On the one hand, string theories naturally lead to an effective scalar-tensor gravity with a running of the parameter $\eta_{g}$ from an initial value $\eta_{g}^{i}$ of order unity. This initial amplitude of violation is essentially preserved during the radiation era since the parameter $\eta_{g}$ depends on the auxiliary scalar field(s) of gravitation, which is(are) frozen during that period. A large SEP violation at recombination should therefore be expected in that context. The order of magnitude of our bounds on $\eta_{g}^{*}$ are still compatible with such a smooth running of that value until recombination time. Improved measurements could however rapidly reveal new physics beyond general relativity.
On the other hand, the experimental constraints at our epoch $\left(\eta_{g}^{0} \leq 1 \times 10^{-3}\right)$ require a strong decrease of $\eta_{g}$ between recombination and today. An attractor mechanism has been advocated for a particular class of scalar-tensor theories, according to which the scalar coupling of gravitation, and consequently the parameter $\eta_{g}$, vanish at late times, to recover general relativity [51]. In this scenario our bounds on the SEP are naturally compatible with the present experimental limits.

\section{CONCLUSION}

The SEP is an essential feature of the theory of general relativity, distinguishing it from any other (experimentally viable) metric theory of gravitation. A violation of the SEP introduces a cosmological Nordtvedt effect in the establishment of the acoustic oscillations imprinted in the CMB temperature power spectrum. The corresponding peaks height therefore measures a gravitational baryonic mass density of the universe. The modified theory considered here introduces this effect as the only signature beyond general relativity, orthogonal to the variation of other cosmological parameters. In this framework we derived constraints on a possible SEP violation, testing in this way Einstein's theory of gravitation, through two independent measurements of the inertial baryonic mass density of the universe. The CMB temperature power spectrum peaks location and the light element abundances in standard BBN respectively lead to $\left|\eta_{g}^{*(C M B)}\right| \leq 0.6$ and $\eta_{g}^{*(B B N-D)}=-0.3 \pm 1$.

More accurate bounds should be determined through a best fit of our modified theory with the experimental data. We also emphasized that, in specific alternatives to general relativity, the cosmological Nordtvedt effect is not the only new effect and the corresponding bounds will in principle be affected. Finally, our approach also offers a possibility of understanding apparent discrepancies between $\mathrm{CMB}$ and $\mathrm{BBN}$ baryon density measurements in terms of new physics.

\section{Acknowledgments}

The authors wish to thank A. Kosowsky, P. J. E. Peebles and N. Sugiyama for interesting comments and discussions. The work of V. B. and J.-M. G. was supported by the Belgian Science Policy through the Interuniversity Attraction Pole P5/27. Y. W. also acknowledges support of the european Harmonic Analysis and Statistics for Signal and Image Processing research network.
[1] L. Page et al., Astrophys. J. Suppl. Ser. 148, 233 (2003).

[2] D. N. Spergel et al., Astrophys. J. Suppl. 148, 175 (2003).
[3] F. R. Bouchet, Preprint astro-ph/0401108 (2004).

[4] P. Coles, P. Dinnen, J. Earl, and D. Wright, Preprint 
astro-ph/0310252 (2003).

[5] F. K. Hansen, A. J. Banday, and K. M. Górski, Preprint astro-ph/0404206 (2004).

[6] A. Hajian and T. Souradeep, Astrophys. J. Lett. 597, L5 (2003).

[7] X. Chen and M. Kamionkowski, Phys. Rev D 60, 104036 (1999).

[8] R. Catena, N. Fornengo, A. Masiero, M. Pietroni, and F. Rosati, Preprint astro-ph/0403614 (2004).

[9] R. Nagata, T. Chiba, and N. Sugiyama, Phys. Rev. D 69, 083512 (2004).

[10] R. Nagata, T. Chiba, and N. Sugiyama, Phys. Rev. D 66, 103510 (2002).

[11] V. Boucher, J.-M. Gérard, P. Vandergheynst, and Y. Wiaux, Preprint astro-ph/0407508 (2004).

[12] J.-M. Gérard and Y. Wiaux, Phys. Rev. D 66, 024040 (2002).

[13] T. Damour and G. Esposito-Farèse, Class. Quantum Grav. 9, 2093 (1992).

[14] K. Nordtvedt, Phys. Rev. 169, 1014 (1968).

[15] K. Nordtvedt, Phys. Rev. 169, 1017 (1968).

[16] C. M. Will, Living Rev. Rel. 4, 4 (2001).

[17] J. G. Williams, S. G. Turyshev, and T. W. Murphy Jr., Int. J. Mod. Phys. D 13, 567 (2004).

[18] M. Bailes, S. M. Ord, H. S. Knight, and A. W. Hotan, Astrophys. J. Lett. 595, L49 (2003).

[19] V. M. Kaspi et al., Astrophys. J. 543, 321 (2000).

[20] G. Esposito-Farèse, Preprint gr-qc/0402007 (2004).

[21] W. Hu, M. Fukugita, M. Zaldarriaga, and M. Tegmark, Astrophys. J. 549, 669 (2001).

[22] W. Hu and M. White, Astrophys. J 471, 30 (1996).

[23] W. Hu and N. Sugiyama, Phys. Rev. D 51, 2599 (1995).

[24] W. Hu and N. Sugiyama, Astrophys. J. 444, 489 (1995).

[25] W. Hu, Ph.D. Thesis, UC Berkeley, Preprint astro-ph/9508126 (1995).

[26] A. Kosowsky, M. Milosavljevic, and R. Jimenez, Phys. Rev. D 66, 063007 (2002).

[27] S. M. Carroll and M. Kaplinghat, Phys. Rev. D 65, 063507 (2002).

[28] T. Damour and B. Pichon, Phys. Rev. D 59, 123502 (1999).
[29] Y. I. Izotov and T. X. Thuan, Astrophys. J. 602, 200 (2004).

[30] V. Luridiana, A. Peimbert, M. Peimbert, and M. Cerviño, Astrophys. J. 592, 846 (2003).

[31] B. D. Fields and K. A. Olive, Astrophys. J. 506, 177 (1998).

[32] A. Ford et al., Astron. Astrophys. 393, 617 (2002).

[33] P. Bonifacio et al., Astron. Astrophys. 390, 91 (2002).

[34] S. G. Ryan, T. C. Beers, K. A. Olive, B. D. Fields, and J. E. Norris, Astrophys. J. Lett. 530, L57 (2000).

[35] R. H. Cyburt, B. D. Fields, and K. A. Olive, Phys. Lett. B 567, 227 (2003).

[36] R. H. Cyburt, Preprint astro-ph/0401091 (2004).

[37] A. Coc, E. Vangiono-Flam, P. Descouvemont, A. Adahchour, and C. Angulo, Astrophys. J. 600, 544 (2004).

[38] A. Cuoco et al., Preprint astro-ph/0307213 (2004).

[39] D. Kirkman, D. Tytler, N. Suzuki, J. M. O'Meara, and D. Lubin, Astrophys. J. Suppl. Ser. 149, 1 (2003).

[40] J. M. O'Meara et al., Astrophys. J. 552, 718 (2001).

[41] S. D'Odorico, M. Dessauges-Zavadsky, and P. Molaro, Astron. Astrophys. 368, L21 (2001).

[42] M. Pettini and D. V. Bowen, Astrophys. J. 560, 41 (2001).

[43] T. M. Bania, R. T. Rood, and D. S. Balser, Nature 415, 54 (2002).

[44] N. H. M. Crighton, J. K. Webb, R. F. Carswell, and K. M. Lanzetta, Mon. Not. R. Astron. Soc. 345, 243 (2003).

[45] V. Barger, J. P. Kneller, H.-S. Lee, D. Marfatia, and G. Steigman, Phys. Lett. B 566, 8 (2003).

[46] C. J. Copi, A. N. Davis, and L. M. Krauss, Phys. Rev. Lett. 92, 171301 (2004).

[47] J. P. Kneller and G. Steigman, Phys. Rev. D 67, 063501 (2003).

[48] E. Massó and F. Rota, Preprint astro-ph/0406660 (2004).

[49] J. D. Barrow and R. J. Scherrer, Preprint astro-ph/0406088 (2004).

[50] K. Ichikawa, M. Kawasaki, and F. Takahashi, Preprint astro-ph/0402522 (2004).

[51] T. Damour and K. Nordtvedt, Phys. Rev. Lett. 70, 2217 (1993). 